\title{
As abelhas (Hymenoptera:Apidae) e as plantas fontes de seus recursos em uma área de cerrado da Chapada Diamantina
}

\author{
Guilherme Pereira de Jesus ${ }^{1}$; Cândida Maria Lima Aguiar² \\ 1. Bolsista PROBIC/UEFS, Graduando em Bacharelado em Ciências Biológicas, Universidade Estadual de Feira de \\ Santana, e-mail: guidspj@gmail.com \\ 2. Orientador, Departamento de nome, Universidade Estadual de Feira de Santana, e-mail: \\ candida.aguiar@gmail.com
}

PALAVRAS-CHAVE: insetos; nicho; savana.

\section{INTRODUÇÃO}

Abelhas participam de interações mutualísticas com plantas, onde as primeiras obtém recursos necessários para sua sobrevivência e as plantas tem acesso a vetores para a dispersão de seus gametas masculinos, e consequente maximização da hetorozigose (Roubik, 1989). As necessidades de uma população constituem o nicho, definido por Hutchinson como um hipervolume n-dimensional, onde as dimensões são condições ambientais e recursos, definindo os requerimentos para uma população de uma espécie persistir (Laroca, 1995). Os recursos alimentares usados pelas populações de abelhas apídeas podem ser pólen, nectar ou óleo (Roubik, 1989).

Pesquisas em cerrado são importantes, pois trata-se de um bioma que cobre grande extensão do Brasil, estando sob uma diversidade de condições refletidas em grande variabilidade fitofisionômica e climática. No entanto, trabalhos realizados nesse bioma sobre comunidades de abelhas e os recursos florais que utilizam concentram-se na região Sudeste do Brasil, especialmente nos estados de São Paulo (Pedro et al., 1991; Andena, 2005; Nogueira-Ferreira et al., 2007) e Minas Gerais (Silveira, 1989; Pereira, 2015). Alguns levantamentos foram feitos na região central do bioma Cerrado (Vieira et al., 2008; Lotchenko, 2014). Há grande lacuna no conhecimento cientifico sobre os recursos alimentares usados pelas abelhas nas porções de Cerrado que se estendem pelo Nordeste do Brasil, com poucos estudos realizados em enclaves de cerrado na Chapada Diamantina (Martins, 1995). Diante dessa escassez de informações, pretendeu-se realizar um levantamento sobre as fontes de recursos florais usadas pelas populações de abelhas em uma área de cerrado na Chapada Diamantina, Bahia, bem como analisar a amplitude dos nichos das espécies mais abundantes e os níveis de sobreposição dos nichos tróficos entre estas espécies de abelha.

\section{MATERIAL E MÉTODOS}

A área amostrada consistiu em três pontos de amostragem em vegetação de Cerrado no município de Palmeiras, Chapada Diamantina, $\left(12^{\circ} 41^{\prime} 66^{\prime}\right.$ ' $\mathrm{S}, 41^{\circ} 48^{\prime}$ 33.3' W). As coletas foram feitas bimensalmente, entre abri1/2015 e outubro/2015, janeiro a junho de 2016. O esforço amostral consistiu em 128 horas, com chegada e saída de campo $8 \mathrm{~h}$ ou $9 \mathrm{~h}$ e $16 \mathrm{~h}$, respectivamente, durante dois dias consecutivos. Neste período, três subáreas foram amostradas por um ou dois coletores utilizando rede entomológica. Três transectos para cada área foram demarcados previamente, sendo sorteados um por área para amostragem em cada expedição. Estes foram percorridos pelos coletores, que permaneciam até 10 minutos em cada planta florida, capturando abelhas que pousavam nas flores. Um banco de dados contendo informações sobre as espécies de abelhas coletadas e as plantas visitadas foi criado e alimentado.

A amplitude e sobreposição dos nichos foram analisadas apenas para espécies de abelhas representadas por no mínimo cinco indivíduos, objetivando a redução dos efeitos estocásticos sobre as análises. A amplitude de nicho de cada espécie foi 
mensurada através do índice de Shannon $\mathrm{H}^{\prime}=-\Sigma(\mathrm{pk} x \ln \mathrm{pk})$, onde pk é a proporção de indivíduos da espécie de abelha coletada na planta $\mathrm{k}$ e ln é o logarítmo neperiano do valor pk. (Ludwig \& Reynolds, 1988). A sobreposição de nicho foi calculada utilizando o índice de Schoener (1968), NOih $=1-1 / 2 \Sigma \mid$ pik - phk|, em que pi e ph são as proporções de indivíduos das espécies i e $\mathrm{h}$

coletados em cada planta $(\mathrm{k})$ das espécies i e h, respectivamente. Seu resultado varia de 0 a 1, representando a mais baixa e mais alta sobreposição entre o par de espécies, respectivamente. Apesar da existência de outros índices, este permite a comparação com outros trabalhos sobre o tema que também o utilizaram, como Aguiar (2013).

\section{RESULTADOS E DISCUSSÃO}

Houve 31 espécies de abelhas apídeas em uma amostra de 200 indivíduos pertencentes a 20 gêneros.

O nicho trófico de seis espécies de abelhas foi analisado, cujas visitas foram distribuídas em 15 espécies de plantas, pertencentes a oito famílias, com o predomínio das abelhas altamente eussociais Apis mellifera, Trigona spinipes e Partamona (Tabela 1). Trigona spinipes teve o maior número de visitas (15 espécies), enquanto Apis mellifera concentrou o forrageio em duas plantas e utilizou outras seis espécies de modo periférico. Similar distribuição de abelhas africanizadas entre espécies vegetais foi encontrada por Martins (2003), Andena (2005) e Nogueira-Ferreira (2007) em cerrado.

A sobreposição de nicho foi baixa em todos os pares de espécies comparados (< $30 \%$ ) (Tabela 2). Entre as abelhas eussociais, a sobreposição foi levemente mais alta, com somente a Trigona spinipes estando presente nas fontes de recursos visitadas pelas solitárias (Tabela 1). A semelhança no uso de recursos entre as espécies eussociais também foi observada por Martins (2008) em caatinga. A similaridade na escolha das fontes pode ter como critério a busca por recursos em abundância, sugerindo comportamento generalista e oportunista para as três espécies. Tais tendências também são evidenciadas pela relativamente alta densidade floral da Serjania sp. e Lamiaceae, além da distribuição das espécies de abelhas meliponinas e de Apis mellifera entre variadas fontes de recurso. $\mathrm{O}$ comportamento generalista pode estar relacionado à alta demanda energética decorrente das grandes e perenes colônias que sustentam (Roubik, 1989). Além disso, a menor necessidade de deslocamento proporcionada pelo adensamento das flores e a maior facilidade de recrutamento (Proctor et al., 1996) reduz alguns dos riscos inerentes ao forrageamento (Real, 1986).

As duas espécies do gênero Centris foram coletadas em maior proporção em plantas do gênero Byrsonima sericea (Malpighiaceae) (Tabela 1), refletindo na sobreposição encontrada $(0,29)$. É bem conhecido o hábito desses apídeos de coletar pólen e óleo floral em plantas desta família, o último utilizado na construção dos ninhos e alimentação das larvas (Alves dos Santos, 2009). A sugerida associação é corroborada por vários estudos, dentre eles Aguiar et al. (2017), que encontraram correlação entre abundância de Byrsonima e abundância de algumas espécies de Centris. 
Tabela 1 - Espécies de abelhas mais abundantes e plantas visitadas em uma savana do município de Palmeiras, Chapada Diamantina, Bahia, Brazil.

\begin{tabular}{|c|c|c|c|}
\hline $\begin{array}{l}\text { Espécies de } \\
\text { abelha }\end{array}$ & $\mathbf{N}$ & $\mathbf{H}^{\prime}$ & Plantas visitadas \\
\hline $\begin{array}{l}\text { Apis } \\
\text { mellifera }\end{array}$ & 77 & 1,64 & $\begin{array}{l}\operatorname{Serj}(18) \operatorname{Mim}(10) \operatorname{La}(23) \operatorname{Mik}(2) \operatorname{Stig}(1), \operatorname{Sti}(1) \text {, } \\
\operatorname{Leg}(1), \operatorname{Ma}(1), ?(20)\end{array}$ \\
\hline $\begin{array}{l}\text { Trigona } \\
\text { spinipes }\end{array}$ & 31 & 2,43 & $\begin{array}{l}\operatorname{Sen} 1(8), \operatorname{Malp}(1), \operatorname{Pm}(1), \operatorname{Bs}(1), \operatorname{Mc}(3) \operatorname{Sm}(2) \\
\operatorname{Ast}(1), \operatorname{Tu}(1), \operatorname{La}(1), \operatorname{My}(1), \operatorname{Mim}(1), \operatorname{Stig}(1), \\
\operatorname{Pas}(1), \operatorname{Cr}(1), \operatorname{Ja}(1)\end{array}$ \\
\hline $\begin{array}{l}\text { Partamona } \\
\text { sp. }\end{array}$ & 27 & 1,43 & $\operatorname{Sa}(13) \operatorname{Ast}(8), \operatorname{Serj}(3) \mathrm{Tu}, \operatorname{Pm}(2) \operatorname{Ery}(1)$ \\
\hline $\begin{array}{l}\text { Centris } \\
\text { fuscata }\end{array}$ & 7 & 0,96 & $\mathrm{Ch}$ (4) Bs (2) Ma(1) \\
\hline Centris sp. & 8 & 1,26 & $\mathrm{Ch}(3), \mathrm{Bs}(3) \mathrm{Ma}(1) \mathrm{Cr}(1)$ \\
\hline Ceratina & 8 & 1,21 & $\mathrm{Tu}(4) \operatorname{Cr}(2) \mathrm{My}(1), \mathrm{Bo}(1)$ \\
\hline Total & 158 & & \\
\hline
\end{tabular}

Siglas: Serj=Serjania, Mim=Mimosoidae, La=Lamiaceae, Mik=Mikania, Stig=Stigmaphyllon, Sti=Stilosanthes, Leg=Leguminosae, $\mathrm{Ma}=$ Malvaceae, ?=indeterminada, Sen1=Senna sp.1, Malp=Malphigiaceae, Pm=Periandra mediterranea, Bs=Byrsonima sericea, $\mathrm{Mc}=$ Mycrostacys corniculata, $\mathrm{Sm}=$ Senna macantlera, Ast=Asteraceae, Tu=Turneraceae, Ery=Erythroxylaceae, Ch=Chamaecrista, $\mathrm{Cr}=$ Croton, $\mathrm{My}=\mathrm{Myrtaceae}, \mathrm{Bo}=$ Boraginaceae

Tabela 2 - Sobreposição no uso de recursos alimentares entre abelhas em savana do município de Palmeiras na Chapada Diamantina, Bahia, Brasil.

\begin{tabular}{l|lllll}
\hline Espécies & Partamona & $\begin{array}{l}\text { Apis } \\
\text { mellifera }\end{array}$ & $\begin{array}{l}\text { Centris } \\
\text { fuscata }\end{array}$ & $\begin{array}{l}\text { Centris } \\
\text { sp. }\end{array}$ & Ceratina \\
\hline Trigona spinipes & 0,16 & 0,08 & 0,19 & 0,13 & 0,09 \\
& & 0,10 & 0 & 0 & 0,07 \\
Partamona & & & 0 & 0 & 0 \\
Apis mellifera & & & & 0,29 & 0 \\
Centris fuscata & & & & & 0 \\
Centris sp. & & & & & \\
\hline
\end{tabular}

\section{CONCLUSÃO}

Espécies de abelhas conhecidamente generalistas (espécies eussociais) apresentaram maior largura de nicho, utilizando maior diversidade de recursos do que as espécies menos generalistas, ou especialistas na coleta de recursos específicos, como as espécies solitárias coletoras de óleo. Houve baixa sobreposição de nicho entre as 
espécies, a qual foi maior entre espécies eussociais e as do gênero Centris. Recomendase estudos sobre o conteúdo polínico nas escopas e ninhos das abelhas Apidae, de modo a reduzir as interpretações errôneas predispostas pelo uso exclusivo de coleta em flor.

\section{REFERÊNCIAS}

AGUIAR, C. M. L.; Santos, G. M. D. M.; Martins, C. F.; Presley, S. J. (2013) Trophic niche breadth and niche overlap in a guild of flower-visiting bees in a Brazilian dry forest. Apidologie, v. 44(2), 153-162.

ALVES-DOS-SANTOS, I.; Machado, I. C.; Gaglianone, M. C. (2009). Natural history of the oil-collecting bees. Oecologia Australis, v. 11(4), 544-557.

AGUIAR, C. M. L.; Lua, S.; Silva, M.; Peixoto, P. E. C.; Alvarez, H. M.; de Mendonça Santos, G. M. (2017). The Similar Usage of a Common Key Resource Does Not Determine Similar Responses by Species in A Community of Oil-collecting Bees. Sociobiology, 64(1), 69-77.

ANDENA S. E.; Bego L. R.; Mechi M. R. (2005). A comunidade de abelhas (Hymenoptera, Apoidea) de uma área de cerrado (Corumbataí, SP) e suas visitas às flores. Rev. Bras. Zoocienc 7, 55-91.

LAROCA, S. (1995). Ecologia: Princípios e métodos. Rio de Janeiro: Vozes, 197.

LUDWIG, J.A.; REYNOLDS, J.F. (1988). Statistical ecology, 337.

LOTCHENKO, J. C. P.; Junior, V. V. A.; Rocha, L. P.; Lima, F.; De Oliveira, V. (2014). Estrutura e Composição da Comunidade de Abelhas (Hymenoptera, Apoidea) em uma Região de Cerrado em Dourados (MS). Cadernos de Agroecologia, 9(4).

MARTINS, C. F.; Moura, A. C. D. A.; De Vasconcellos Barbosa, M. R. (2008). Bee plants and relative abundance of corbiculate apidae species in a brazilian caatinga area. Revista Nordestina de Biologia, 17(1), 63-74.

MARTINS, C. F. (1995). Flora apícola e nichos tróficos de abelhas (Hymenoptera:Apoidea) na Chapada Diamantina (Lençóis-BA, Brasil). Revista Nordestina de Biologia 10(4), 119-140.

NEFF, J.L.; Simpson, B. B. (1981). Oil-collecting structures in the Anthophoridae (Hymenoptera): morphology, function, and use in systematics. J our. Ka nsas Ent. Soe. 54 (1), 95-123.

NOGUEIRA-FERREIRA, F. H.; AUGUSTO, S. C. (2007). Amplitude de nicho e similaridade no uso de recursos florais por abelhas eussociais em uma área de cerrado. Bioscience Journal 23(1), 45-51.

PEDRO, S. R.; Camargo, J. M. F. (1991). Interactions on floral resources between the Africanized honey bee Apis mellifera L and the native bee community (Hymenoptera: Apoidea) in a natural "cerrado" ecosystem in southeast Brazil. Apidologie (22),397-415. PEREIRA, S. A. N. (2015). Levantamento da fauna de abelhas no município de Monte Carmelo-MG. Revista Getec, 4(7), 11-24.

POLATTO, L. P.; Alves Jr, V. V. (2008). The use of floral resources by Visitors on Sparattosperma leucanthum (Vell.) K. Schum.(Bignoniaceae). Neotropical entomology, 37(4), 389-398.

PROCTOR, M.; Yeo, P.; Lack, A. (1996). The natural history of pollination. HarperCollins Publishers.

REAL, L.; Caraco, T. (1986). Risk and foraging in stochastic environments. Annual Review of Ecology and Systematics, 17(1), 371-390.

ROUBIK, D. W. (1989). Ecology and natural history of tropical bees. Cambridge University Press, Crambidge.

VIEIRA, G. H. D. C.; Marchini, L. C.; Souza, B. D. A.; Moreti, A. C. D. C. C. (2008). Floral sources used by bees (Hymenoptera, Apoidea) in a savannah area of Cassilândia county, Mato Grosso do Sul, Brazil. Ciência e Agrotecnologia, 32(5), 1454-1460. 

